\title{
Nonlinear predictive high order sliding mode control for permanent magnet synchronous motor drive system
}

\author{
Xudong Liu, Chenghui Zhang*, Ke Li, Qi Zhang \\ School of Control Science and Engineering, Shandong University, Jinan, China.
}

\begin{abstract}
The speed control problem for permanent magnet synchronous motor (PMSM) drive system is studied in this paper. The predictive control has been proved as an effective solution for the fast transient response, but the performance will be deteriorated in the presence of model uncertainties and load disturbance. Thus a composite control method combining the nonlinear generalized predictive control (GPC) with the sliding mode control is proposed. In view of the chattering problems caused by the traditional sliding mode control, a high order terminal sliding mode controller is designed, which can reduce the chattering effectively. In the end, the stability of the system is proved. The simulation and experimental results show that, compared with PI controllers, the designed controller has the faster speed response and the stronger robustness, and the chattering is decreased obviously than the first order sliding mode controller. (c)2016 All rights reserved.
\end{abstract}

Keywords: Permanent magnet synchronous motor, generalized predictive control, high order terminal sliding mode control.

2010 MSC: 05C12, 05C15, 05C76.

\section{Introduction}

Due to high efficiency, high power density and good reliability, permanent magnet synchronous motor (PMSM) drive system is intensively used in industrial applications [9]. The field orientation vector control strategy is usually employed to control PMSM. The technology makes PMSM achieve similar control performance as a DC motor. For the vector control, proportional plus integral (PI)

${ }^{*}$ Corresponding author

Email address: zchui@sdu.edu.cn (Chenghui Zhang) 
control method, which is independent of the mathematical model, was popular in the past. Although this control method has a simple structure and robustness, it may still fail to meet the high performance requirement with fast response and strong robustness in the practical applications. To improve the motor performance, quite a few approaches such as, back-stepping control [17], model predictive control [12], active disturbance rejection control [13] and sliding mode control [15], have been put forward based on modern control theories, which enhance the motor control performance in different aspects.

Among these approaches, the predictive control appears to be a very interesting alternative method for the drive control system, which offers the potential for achieving the faster transient response, more precise control and full-compatibility with the digital controller. In [12], the predictive function controller is designed for the speed control of PMSM, and extend state observer is introduced to estimate the disturbance, which improves the robustness of the system. In [3], the cascade model predictive control method is studied for the PMSM drive system, and a disturbance model is embedded in the predictive control for the problem of speed fluctuation caused by the periodic disturbance. In [2], a model predictive control algorithm with the single loop control structure is studied. The predictive controllers aforementioned are all designed by the discrete time model. In [7, a nonlinear generalized predictive control (GPC) method based on the continuous time model is studied for the speed control of PMSM. The controller is designed by the Taylor series expansion method, and it needs a little calculation.

However, PMSM is an uncertain system, and it inevitably faces with the disturbance, e.g., load disturbance and model uncertainties. The control performance of the predictive controller may be strongly affected in the presence of model uncertainties and load disturbance. Thus, it is possible to design a composite controller combining the generalized predictive controller with the robust controller, in which, the dynamic response and the robustness can be assured, simultaneously. In the robust control methods, sliding mode control is well-known for its invariant properties to certain internal parameter variations and external disturbances. There are also some cheering research reports about the sliding mode control for PMSM control system. In [16], a variable sliding mode speed controller combined with the exponential reaching law is proposed. In [18], a sliding mode controller based on the novel reaching law is presented, and a sliding mode observer is used to estimate the uncertainties. The terminal sliding mode control method for PMSM is introduced in [11], and the system can achieve the finite time convergence. As we all know, the main disadvantage of the sliding mode control is the inherent chattering problem. In order to reduce the chattering, the boundary layer technology is mainly adopted [1, but it may decrease the robustness of the system.

Recently, the high order sliding mode control has become the interesting approach to eliminate chattering. The key idea of the high order sliding mode control is that both the sliding manifold and its higher order derivatives need to be zero [6, 10]. Through applying the high frequency switching control to the higher order derivatives of the sliding mode variable, the chattering may be eliminated completely, meanwhile, the robustness is unaffected. Based on the high order sliding mode control theory, the speed controller for PMSM is designed in [8, 14], and the results show that the chattering can be reduced effectively. But in the sliding mode controller, due to the reason that the system has good robustness only on the sliding mode surface, to bring the system onto the sliding surface, a very high control input may be needed, which will influence the dynamic performance of the drive system.

In this paper, a generalized predictive control method combining with the high order sliding mode control is proposed for the speed controller of PMSM. Firstly, the generalized predictive control using the continuous time model is introduced for the nominal motor drive system through the Taylor series expansion. The controller can achieve fast speed response which is easily achieved. Then, considering 
the model uncertainties and load disturbance, the high order terminal sliding mode controller based on the predictive controller is designed, which can compensate the influence of the disturbance, and the robustness is improved effectively. The motivation of the paper is attempting to use the merits of predictive control and the high order terminal sliding mode control, and the fast transient response and the strong robustness are realized. In addition, the chattering is obviously reduced. The proposed method is firstly applied in the PMSM drive system. In the end, the simulation and experiment demonstrate the validity of the designed speed controller for PMSM drive system.

This paper proceeds as follows. Section 2 defines the mathematical model of PMSM. Section 3 presents the design process of the speed controller. The simulation and experiment are given in Section 4, which is followed by conclusion in Section 5 .

\section{The mathematical model of PMSM}

According to the method of rotor field oriented, the mathematical model of PMSM can be described in synchronously rotating frame as

$$
\begin{aligned}
& \left\{\begin{array}{l}
u_{d}=L_{d} \frac{d i_{d}}{d t}+R_{s} i_{d}-n_{p} \omega L_{q} i_{q}, \\
u_{q}=L_{q} \frac{d i_{q}}{d t}+R_{s} i_{q}+n_{p} \omega L_{d} i_{d}+n_{p} \omega \Phi,
\end{array}\right. \\
& J \frac{d \omega}{d t}=n_{p}\left[\left(L_{d}-L_{q}\right) i_{d} i_{q}+\Phi i_{q}\right]-\tau_{L}-b \omega,
\end{aligned}
$$

where $L_{d}$ and $L_{q}$ are the stator inductance, $i_{d}$ and $i_{q}$ are the stator current, $u_{d}$ and $u_{q}$ are the stator input voltage in $d q$ rotating frame. $R_{s}$ is the per-phase stator resistance, $n_{p}$ is the number of pole pairs, $\omega$ is the mechanical angular speed of the rotor, $\Phi$ is the rotor flux. $J$ is the inertia, $\tau_{L}$ is the load torque, and $b$ is the viscous friction coefficient.

The general PMSM control system structure investigated in this work is shown in Figure 1. A structure of cascade control, which includes the speed loop and the current loop, is employed. In this paper, the generalized predictive high order sliding mode controller is adopted in the speed controller to track the reference speed. The $d$-axes reference current is set to $i_{d}^{*}=0$ and $q$-axes reference current $i_{q}^{*}$ is the output of the speed controller.

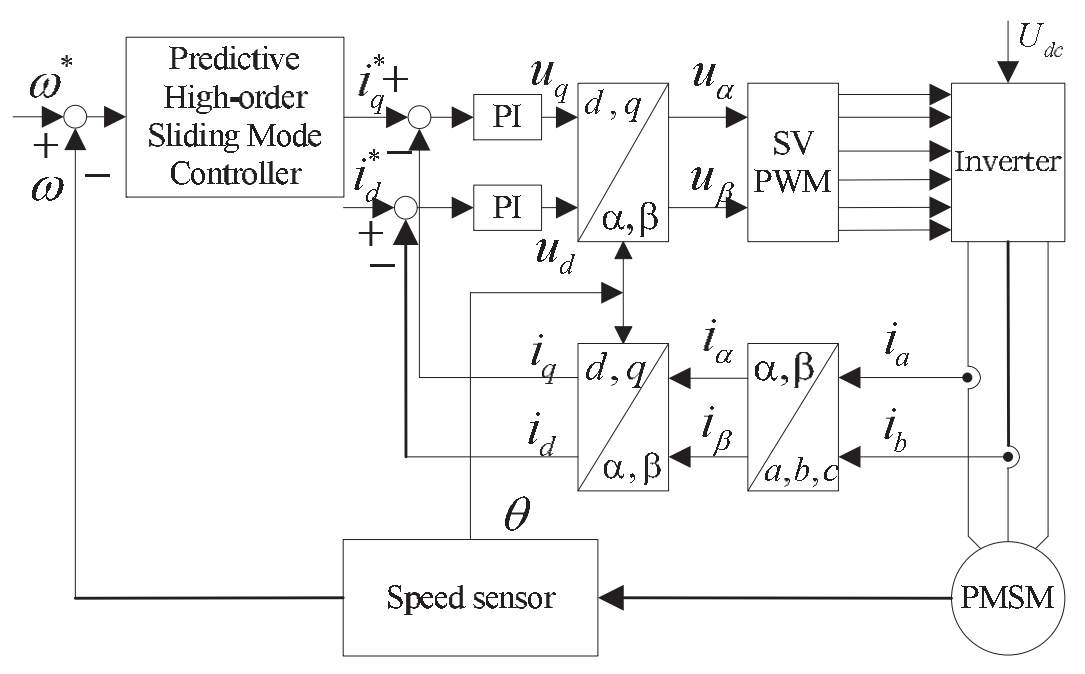

Figure 1: The structure of the PMSM control system. 


\section{The design of speed controller for PMSM}

The control objective is to find a controller $i_{q}^{*}$ that assures internal stability and realize the fast speed tracking response of PMSM, and the controller should have the strong robustness for the disturbance, simultaneously. A composite controller based on the generalized predictive control and high order sliding mode control is studied. The controller $i_{q}^{*}$ is designed as $i_{q}^{*}=i_{q 1}+i_{q 2}$, where the predictive controller $i_{q 1}$ is designed for the nominal part of the system without considering the disturbance, and the sliding mode controller $i_{q 2}$ is added to the predictive controller to improve the robustness. The structure of the speed controller is shown in Figure 2.

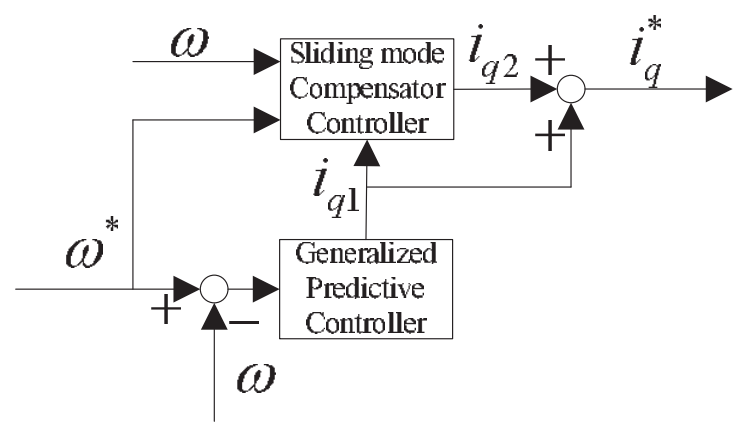

Figure 2: The structure of the speed controller.

\subsection{The design of generalized predictive controller}

The generalized predictive control method [4] based on the continuous time model will be introduced for the speed controller. Define $\omega^{*}$ as the reference motor speed, the state variable is $x=\omega$, the input variable $u_{1}=i_{q 1}$, the output variable $y=h(x)=\omega$, and the disturbance $d$ includes the model uncertainties and the load torque. Because $i_{d}^{*}=0$ is used in the controller, the item $n_{p}\left(L_{d}-L_{q}\right) i_{d} i_{q}$ in $(2.1)$ is approximately equal to zero. Then the nominal part of system model $(2.1)$ without considering the disturbance can be expressed as

$$
\left\{\begin{array}{l}
\dot{x}=f(x)+g_{1} u_{1}, \\
y=h(x)=g_{2} x,
\end{array}\right.
$$

where $f(x)=-\frac{b}{J} \omega, g_{1}=\frac{n_{p} \Phi}{J}, g_{2}=1$. First of all, define the cost function as

$$
J_{a}=\frac{1}{2} \int_{0}^{T_{r}}\left(\hat{\omega}(t+\tau)-\omega^{*}(t+\tau)\right)^{2} d \tau
$$

where $T_{r}$ is the predictive horizon. $\hat{\omega}(t+\tau)$ and $\omega^{*}(t+\tau)$ represent the predictive speed and the reference speed, respectively.

The input relative degree $\rho$ of the outputs is equal to 1 . Hence, the Taylor series expansion yields

$$
\hat{\omega}(t+\tau)=\Gamma(\tau) \bar{W}(t)
$$

where $\Gamma(\tau)=\left[\begin{array}{ll}1 & \tau\end{array}\right], \bar{W}(t)=[\hat{\omega}(t) \dot{\hat{\omega}}(t)]^{T}$. Similarly, the Taylor series expansion of the reference speed is

$$
\omega^{*}(t+\tau)=\Gamma(\tau) \bar{W}^{*}(t)
$$


where $\bar{W}^{*}(t)=\left[\begin{array}{cc}\omega^{*}(t) & \dot{\omega}^{*}(t)\end{array}\right]^{T}$.

Define $\bar{\Gamma}\left(T_{r}\right)=\int_{0}^{T_{r}} \Gamma^{T}(\tau) \Gamma(\tau) d \tau$, and the $(i, j)$-th element in the matrix $\bar{\Gamma}\left(T_{r}\right)$ can be derived as

$$
\bar{\Gamma}\left(T_{r}\right)_{(i, j)}=\frac{1}{(i-1) !(j-1) !(i+j-1)} T_{r}^{i+j-1}, \quad i . j=1,2 .
$$

From 3.2 and 3.3 , the cost function 3.1 can be equal to

$$
J_{a}=\frac{1}{2}\left[\bar{W}(t)-\bar{W}^{*}(t)\right]^{T} \bar{\Gamma}\left(T_{r}\right)\left[\bar{W}(t)-\bar{W}^{*}(t)\right] .
$$

Then, the predictive control law can be derived by minimizing the cost function as

$$
i_{q 1}=-G^{-1}(x)\left(k M_{\rho}+L_{f} h(x)-\dot{\omega}^{*}\right),
$$

where $G(x)=L_{g_{1}} L_{f}^{\rho-1} h(x)=L_{g_{1}} \omega=\frac{n_{p} \Phi}{J}, L_{f} h(x)=-\frac{b}{J} \omega, M_{\rho}=\omega-\omega^{*}$ and $k=\frac{3}{2 T_{r}}$.

It is worth pointing out that the predictive controller is derived based on the nominal model. The stability and the robustness may be affected by the disturbance. For this reason, it is necessary to consider the disturbance to strength the robustness of the speed controller, meanwhile, the fast response is not impacted, which is the focus of the paper.

\subsection{The design of high order sliding mode compensator controller}

In order to improve the robustness of the system, a high order sliding mode compensator controller $i_{q 2}$ is designed in this section. Because of the simplicity and less information demand, a second order sliding mode controller is chosen in the paper. First, define $e=\omega-\omega^{*}$, then $\dot{e}$ can be derived as

$$
\dot{e}=-\frac{b}{J} e+\frac{n_{p} \Phi}{J} i_{q}-\frac{b}{J} \omega^{*}-\dot{\omega}^{*}+d .
$$

Due to the system has robustness to the disturbance only on the sliding mode surface, to ensure the robustness, the motion of the system should be on the sliding mode surface as far as possible [5]. So the sliding mode surface is defined as

$$
s(t)=G\left[e-\int_{0}^{t} \dot{\varphi}(\tau) d \tau\right],
$$

where $G$ is the designed parameter, $\dot{\varphi}(\tau)=-\frac{b}{J} e-\frac{b}{J} \omega^{*}-\dot{\omega}^{*}+\frac{n_{p} \Phi}{J} i_{q 1}$, then

$$
\dot{s}(t)=G\left[\dot{e}+\frac{b}{J} e+\frac{b}{J} \omega^{*}+\dot{\omega}^{*}-\frac{n_{p} \Phi}{J} i_{q 1}\right] .
$$

Substituting 3.4 into 3.5

$$
\begin{aligned}
\dot{s}(t) & =G\left[-\frac{b}{J} e-\frac{b}{J} \omega^{*}-\dot{\omega}^{*}+\frac{n_{p} \Phi}{J} i_{q 1}+\frac{n_{p} \Phi}{J} i_{q 2}+d+\frac{b}{J} e+\frac{b}{J} \omega^{*}+\dot{\omega}^{*}-\frac{n_{p} \Phi}{J} i_{q 1}\right] \\
& =G\left[\frac{n_{p} \Phi}{J} i_{q 2}+d(t)\right]
\end{aligned}
$$

and

$$
\ddot{s}(t)=G\left[\frac{n_{p} \Phi}{J} i_{q 2}+\dot{d}(t)\right]
$$


According to $s(t)$ and $\dot{s}(t)$, a second order nonsingular terminal sliding mode surface is defined as

$$
\sigma(t)=s(t)+\delta \dot{s}(t)^{\alpha / \beta},
$$

where $\delta>0, \alpha, \beta \in 2 n+1, n$ is an integer, and $1<\alpha / \beta<2$. By using the terminal sliding mode control, the system can achieve the finite time convergence [6]. Then

$$
\dot{\sigma}(t)=\dot{s}(t)+\frac{\alpha}{\beta} \dot{s}(t)^{\alpha_{/ \beta}-1} \ddot{s}(t)=\frac{\alpha}{\beta} \delta \dot{s}(t)^{\alpha / \beta^{-1}}\left[\frac{\beta}{\alpha \delta} \dot{s}(t)^{2-\alpha / \beta}+\ddot{s}(t)\right],
$$

when $\dot{s}(t) \neq 0, \dot{s}(t)^{\alpha / \beta^{-1}}>0$ can be guaranteed.

Next, the control input $i_{q_{2}}$ should be designed in such a way that the sliding-mode reaching condition is met. Thus, the equal reaching law is typically chosen as

$$
\dot{\sigma}(t)=-\eta_{1} \operatorname{sgn}(\sigma(t)),
$$

where $\eta_{1}>0$. From $(3.8)$ and $(3.9)$ we get

$$
\frac{\alpha}{\beta} \delta \dot{s}(t)^{\alpha / \beta^{-1}}\left[\frac{\beta}{\alpha \delta} \dot{s}(t)^{2-\alpha / \beta}+\ddot{s}(t)\right]=-\eta_{1} \operatorname{sgn}(\sigma(t)) .
$$

Define $\eta_{2}=\frac{\alpha}{\beta} \delta \dot{s}(t)^{\alpha / \beta^{-1}}, \eta=\frac{\eta_{1}}{\eta_{2}}$, then $\ddot{s}(t)$ can be expressed as

$$
\ddot{s}(t)=-\frac{\beta}{\alpha \delta} \dot{s}(t)^{2-\alpha / \beta}-\eta \operatorname{sgn}(\sigma(t)) .
$$

According to 3.7 and 3.10 , the second order terminal sliding mode controller is designed as

$$
i_{q_{2}}=-\int_{0}^{t} \frac{J}{G n_{p} \Phi}\left[\frac{\beta}{\alpha \delta} \dot{s}(t)^{2-\alpha / \beta}+\eta \operatorname{sgn}(\sigma(t))\right] d \tau,
$$

where $|G \dot{d}(t)|<\eta$ should be satisfied.

\subsection{Robustness analysis of the system}

To prove the stability of the system, we can define the Lyapunov function as $V=\frac{1}{2} \sigma(t)^{T} \sigma(t)$, then

$$
\begin{aligned}
\dot{V} & =\sigma(t) \dot{\sigma}(t) \\
& =\sigma(t)\left[\dot{s}(t)+\frac{\alpha}{\beta} \delta \dot{s}(t)^{\alpha / \beta^{-1}} \ddot{s}(t)\right] \\
& =\sigma(t)\left[\dot{s}(t)+\frac{\alpha}{\beta} \delta \dot{s}(t)^{\alpha / \beta^{-1}}\left(\frac{G n_{p} \Phi}{J} i_{q 2}+G \dot{d}(t)\right)\right] \\
& =\sigma(t)\left[\dot{s}(t)+\frac{\alpha}{\beta} \delta \dot{s}(t)^{\alpha / \beta^{-1}}\left(-\frac{\beta}{\alpha \delta} \dot{s}(t)^{2-\alpha / \beta}-\eta \operatorname{sgn}(\sigma(t))+G \dot{d}(t)\right)\right] \\
& =\sigma(t) \frac{\alpha}{\beta} \delta \dot{s}(t)^{\alpha / \beta^{-1}}(-\eta \operatorname{sgn}(\sigma(t))+G \dot{d}(t)) .
\end{aligned}
$$

Because of $|G \dot{d}(t)|<\eta, \dot{V}<0$ can be derived for $\dot{s}(t) \neq 0$. According to Lyapunov stability theorem, the system is asymptotically stable.

The final speed controller for the PMSM drive system can be designed from (3.1) and (3.11). The designed controller can guarantee the fast speed response and improve the robustness of the system. Simultaneously, the chattering caused by the sliding mode control can be reduced. 


\section{Simulation and experiment}

To demonstrate the effectiveness of the proposed speed controller, simulation and experiment on the PMSM closed loop control system has been performed. The motor parameters are given in Table 1.

Table 1: Parameters of PMSM

\begin{tabular}{ccc}
\hline Description & Value & Unit \\
\hline nominal speed & 3000 & $\mathrm{r} / \mathrm{min}$ \\
nominal torque & 2.3 & $\mathrm{~N} \cdot \mathrm{m}$ \\
resistance & 4.8 & $\Omega$ \\
inertia & 19.5 & $\mathrm{~Wb}$ \\
friction coefficient & 0.0004 & $\mathrm{~N} \cdot \mathrm{m} \cdot \mathrm{s} / \mathrm{rad}$ \\
rotor flux & 0.15 & $\mathrm{~Wb}$ \\
\hline
\end{tabular}

\subsection{Simulation and analysis}

In this section, the simulation of the PMSM drive system is completed by Matlab, and three speed controllers, i.e., PI controller, GPC controller, and the proposed predictive high order terminal sliding mode controller (GPC+HOSMC), are designed and compared. The current controllers are all designed by the PI control method. The sample time is $T_{s}=0.0001 s$, the predictive horizon $T_{r}=0.001 s$. In the HOSMC, $G=0.05, \delta=0.01, \alpha / \beta=3 / 2$. To have a fair comparison, the current loop has the same parameters in each method. The gains of the PI controller are obtained by trial and error so as to achieve satisfactory performance.

In the motor control system, the reference speed is passed through a second order linear filter as $F(s)=\frac{\omega_{n}^{2}}{s^{2}+2 \xi \omega_{n} s+\omega_{n}^{2}}$, where $\xi=1$ and $\omega_{n}=100$ is chosen. The reference speed is given as $1000 \mathrm{r} / \mathrm{min}$. At $t=0.5 \mathrm{~s}$, the disturbance load torque $\tau_{L}=1 \mathrm{~N} \cdot \mathrm{m}$ is added. The corresponding motor speed and current waveforms of PI controller, GPC controller, GPC+SMC controller and the proposed controller are shown in Fig. 3-Fig. 5.

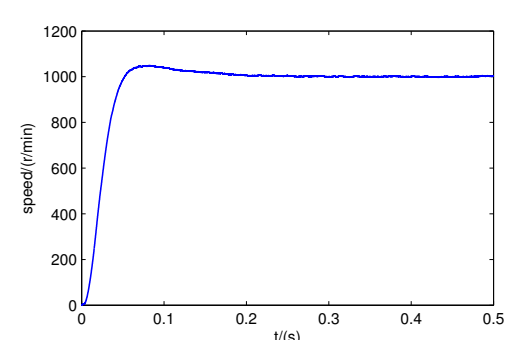

(a)

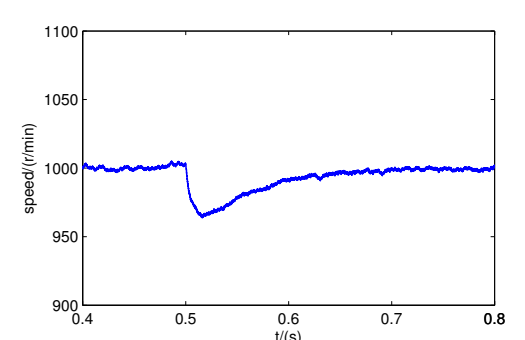

(b)

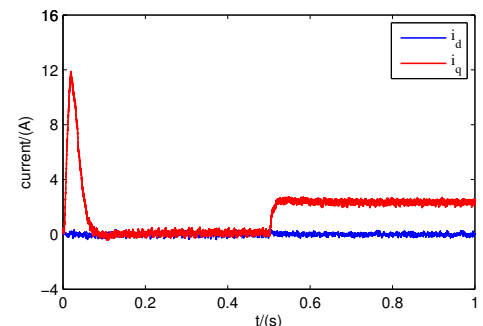

(c)

Figure 3: Simulation results under PI controller: (a) Speed response, (b) Speed variation with load disturbance, (c) $d q$-axes current.

As shown in Fig. 3FFig. 5, when the motor starts, a large starting current is proposed in the motor. Compared with PI controller, the speed controller based on other methods has the fast response and small overshoot. 


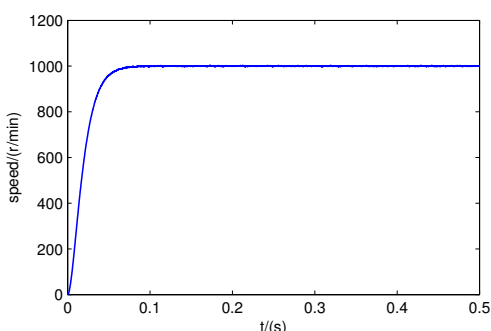

(a)

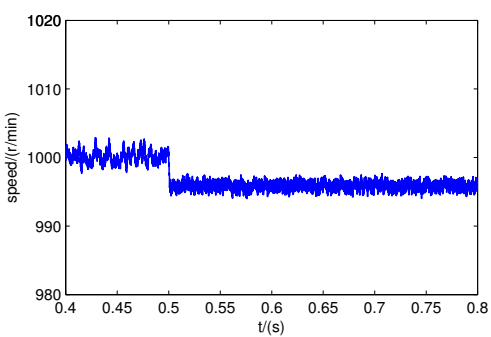

(b)

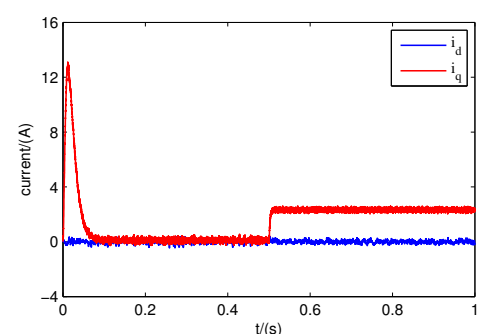

(c)

Figure 4: Simulation results under GPC: (a) Speed response, (b) Speed variation with load disturbance, (c) dq-axes current.

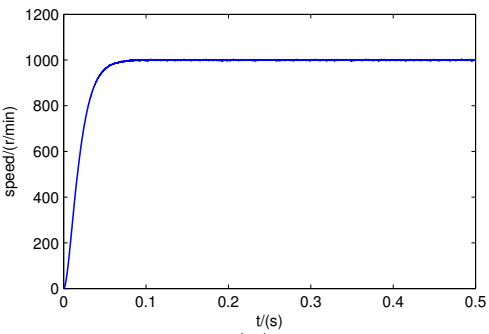

(a)

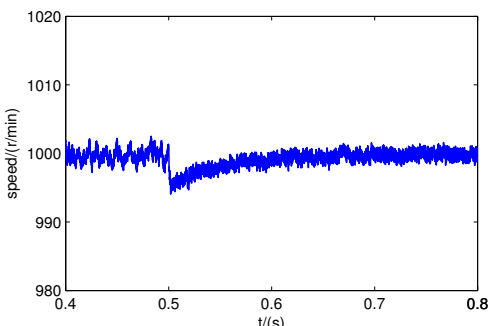

(b)

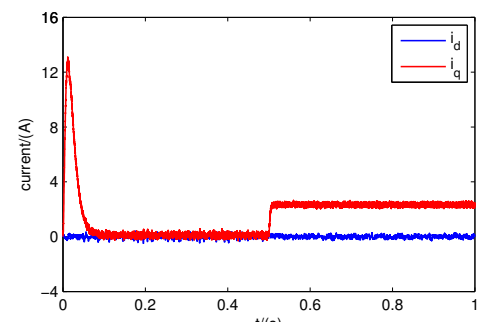

(c)

Figure 5: Simulation results under the proposed controller: (a) Speed response, (b) Speed variation with load disturbance, (c) $d q$-axes current.

When the load torque is added to the system, the GPC controller will have a steady-state error, and the system has the poor robustness. The PI controller and the proposed controller have no steady-state error under the load disturbance. But in the PI controller, the motor speed has a bigger depreciation and recovers to the reference value in a longer time length. The GPC+HOSMC controller has better disturbance rejection ability.

To further illustrate the advantages of the proposed GPC+HOSMC speed control method for PMSM, it is compared with the GPC under the traditional sliding mode controller (GPC+SMC). In this method, the controller is designed from (3.6), the equal reaching law is chosen as $\dot{s}(t)=$ $-\eta \operatorname{sgn}(s(t))$, and the switching gains of SMC and HOSMC are both selected as $\eta=100$. The results are shown in Fig. 6.

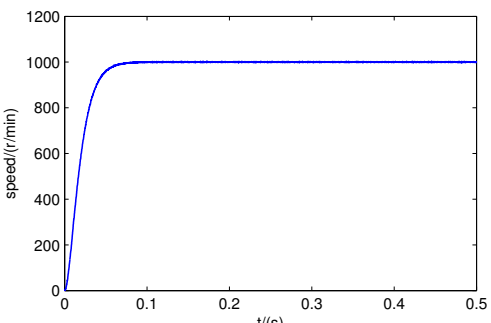

(a)

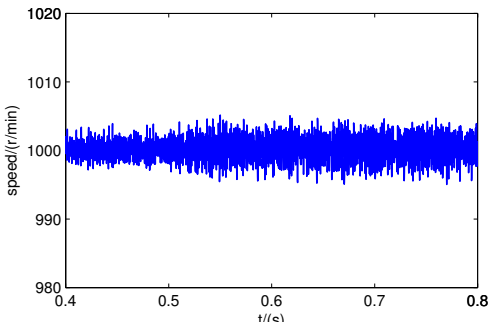

(b)

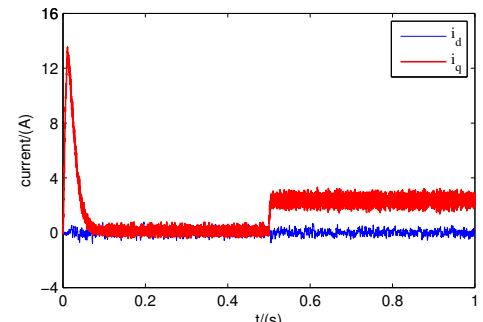

(c)

Figure 6: Simulation results under GPC+SMC: (a) Speed response, (b) Speed variation with load disturbance, (c) $d q$-axes current.

From the speed and current waveforms in Fig. 6, it can be seen that the traditional sliding mode controller has a good transient response, and when the load disturbance is added to the motor, the 
speed has a very small variation. However, the higher chattering exists in the speed and current waveforms. On the basis of the comparison, the high order sliding mode controller has the better steady state performance.

In summary, the results above show that the designed controller has the better transient and steady state performance. It has the strong robustness under parameter uncertainties and load disturbance.

\subsection{Experiment and analysis}

To test the speed control performance of the designed predictive high order terminal sliding mode controller for PMSM, the experimental bed is constructed, which consists of a six-pole 0.72 $\mathrm{kW}$ interior PMSM coupled to a dynamometer, and an IPM based on inverter with dSPACE as a controller is adopted as the driver. In the experiment, the load torque is realized by hysteresis dynamometer, so the excitation current can not be changed suddenly, which will cause a little delay for the load torque. The parameters given in the experiment are: $T_{s}=0.0001 s, T_{r}=0.001 s$, $G=0.05, \delta=0.05, \alpha / \beta=3 / 2, \eta=100$.

The speed controller is designed by the GPC+HOSMC controller. The motor reference speed is given as $1000 \mathrm{r} / \mathrm{min}$, the speed waveform when the motor starts is shown in Fig. 7 (a). After the motor is stable, the disturbance about $1 N \cdot m$ is added to the motor by the dynamometer, and the speed variation waveform is shown in Fig. 7 (b). When the speed is increased from $1000 \mathrm{r} / \mathrm{min}$ to $1500 \mathrm{r} / \mathrm{min}$, the speed response waveform is shown in Fig. 7 (c).

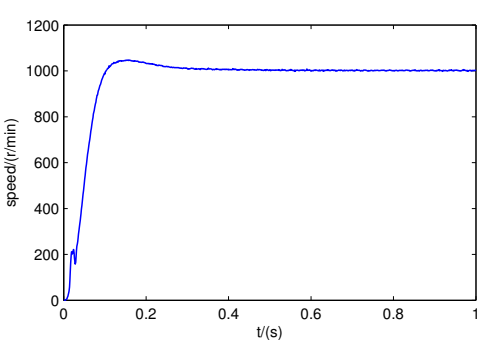

(a)

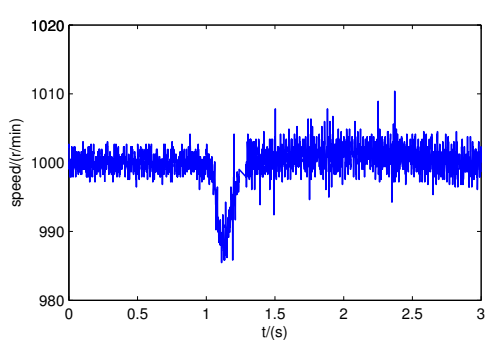

(b)

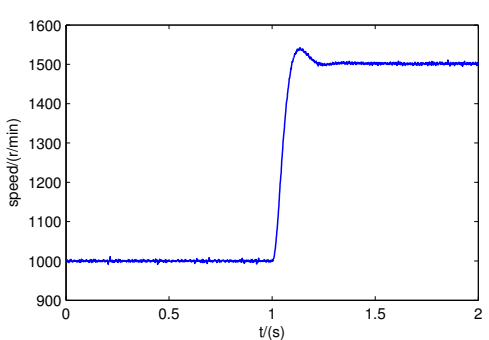

(c)

Figure 7: Experimental results under the GPC+HOSMC controller: (a) Speed response when the motor starts, (b) Speed variation with load disturbance, (c) Speed variation when the reference speed is increased.

As seen in the Fig. 7, when the motor starts, the speed can track the reference speed quickly with a small overshoot, the speed has pleasure steady and transient performance. While a load disturbance about $1 N \cdot m$ is added, a small speed drop is produced about $12 \mathrm{r} / \mathrm{min}$, and the speed can recover to the reference value quickly. When the speed changes, the controller also has good tracking performance. The experimental results above prove that the proposed speed control method has the outstanding steady and dynamic performance no matter the motor has the disturbance, which can be taken as an alternative scheme for PMSM drive system.

\section{Conclusions}

A novel speed control method for PMSM is proposed in the paper. The designed speed controller integrates the advantages of the predictive control and the high order terminal sliding mode control, which has the fast dynamic response and well robust performance. Meanwhile, it has the smaller chattering than the traditional sliding mode controller. By comparing with other control methods, the effectiveness of the designed controller is verified. The designed controller is simple and convenient to be completed. The designed speed controller can also be used in the other motor drive system. 


\section{Acknowledgment}

This work is supported by the National Key Basic Research Problem of China (Grant no. 2013CB035600), the Major International (Regional) Joint Research Project of the National Natural Science Foundation of China (Grant no. 61320106011), the National Natural Science Foundation of China (Grant nos. 51277116, 61304029, 61304033, 61403236).

\section{References}

[1] I. C. Baik, K. H. Kim, M. J. Youn, Robust nonlinear speed control of PM synchronous motor using boundary layer integral sliding mode control technique, IEEE Trans. Control Syst. Technol., 8 (2000), 47-54. 1

[2] S. Bolognani, S. Bolognani, L. Peretti, M. Zigliotto, Design and implementation of model predictive control for electrical motor drives, IEEE Trans. Ind. Electron., 56 (2009), 1925-1936. 1

[3] S. Chai, L. Wang, E. Rogers, A cascade MPC control structure for PMSM with speed ripple minimization, IEEE Trans. Ind. Electron., 60 (2013), 2978-2987. 1

[4] W. H. Chen, D. J. Ballance, P. J. Gawthrop, Optimal control of nonlinear systems: a predictive control approach, Automatica J. IFAC, 39 (2003), 633-641. 3.1

[5] M. Comanescu, L. Xu, T. D. Batzel, Decoupled current control of sensorless induction-motor drives by integral sliding mode, IEEE Trans. Ind. Electron., 55 (2008), 3836-3845. 3.2

[6] M. Das, C. Mahanta, Optimal second order sliding mode control for linear uncertain systems, ISA Trans., 53 (2014), 1807-1815. 1, 3.2

[7] R. Errouissi, M. Ouhrouche, W. H. Chen, A. M. Trzynadlowski, Robust cascaded nonlinear predictive control of a permanent magnet synchronous motor with antiwindup compensator, IEEE Trans. Ind. Electron., 59 (2012), 3078-3088. 1

[8] Y. Huangfu, S. Laghrouche, W. Liu, R. Q. Ma, A. Miraui, Chattering avoidance high order sliding mode control for permanent magnet synchronous motor, Electric Mach. Control, 16 (2012), 7-11. 1

[9] R. Krishnan, Electric motor drives: modeling, analysis, and control, Prentice Hall, New York, (2001). 1

[10] S. Laghrouche, F. Plestan, A. Glumineau, Higher order sliding mode control based on integral sliding mode, Automatica J. IFAC, 43 (2007), 531-537. 1

[11] S. Li, M. Zhou, X. Yu, Design and implementation of terminal sliding mode control method for PMSM speed regulation system, IEEE Trans. Ind. Electron., 9 (2013), 1879-1891. 1

[12] H. Liu, S. Li, Speed control for PMSM servo system using predictive functional control and extended state observer, IEEE Trans. Ind. Electron., 59 (2012), 1171-1183. 1

[13] H. Sira-Ramírez, J. Linares-Flores, C. García-Rodríguez, M. A. Contreras-Ordaz, On the control of the permanent magnet synchronous motor: an active disturbance rejection control approach, IEEE Trans. Control Syst. Technol., 22 (2014), 2056-2063. 1

[14] Y. M. Wang, Y. Feng, Q. L. Lu, Design of free-chattering sliding mode control systems for permanent magnet synchronous motor, Electric Mach. Control, 12 (2008), 514-519. 1

[15] Q. Wang, B. Xu, L. You, X. Wang, BPMSM control using a sliding mode controller with fractional order of suspension forces based on differential geometry, Internat. J. Control Autom., 8 (2015), 187-198. 1

[16] H. Wang, B. Zhou, S. Fang, A PMSM sliding mode control system based on exponential reaching law, Trans. China Electrotechnical Soc., 24 (2009), 71-77. 1

[17] J. Yong-Ho, S. W. Lee, The implementation of speed control on IPMSM using simple nonlinear adaptive back-stepping, Internat. J. Control Autom., 7 (2014), 343-352. 1

[18] X. Zhang, L. Sun, K. Zhao, L. Sun, Nonlinear speed control for PMSM system using sliding-mode control and disturbance compensation techniques, IEEE Trans. Ind. Electron., 28 (2013), 1358-1365. 1 